\title{
STATISTICAL PROPERTIES OF THE ENERGY RELEASE IN EMERGING AND EVOLVING ACTIVE REGIONS
}

\author{
Loukas Vlahos, Tassos Fragos, and Heinz Isliker \\ Department of Physics, University of Thessaloniki, 54006 Thessaloniki, Greece \\ AND \\ MaNOLIS GeORgOUlis \\ Johns Hopkins University, Applied Physics Laboratory, 11100 Johns Hopkins Road, Laurel, MD 20723-6099 \\ Received 2002 April 30; accepted 2002 July 12; published 2002 July 19
}

\begin{abstract}
The formation and evolution of active regions are inherently complex phenomena. Magnetic fields generated at the base of the convection zone follow a chaotic evolution before reaching the solar surface. In this article, we use a two-dimensional probabilistic cellular automaton to model the statistical properties of the magnetic patterns formed on the solar surface and to estimate the magnetic energy released in the interaction of opposite polarities. We assume that newly emerged magnetic flux tubes stimulate the emergence of new magnetic flux in their neighborhood. The flux tubes move randomly on the surface of the Sun, and they cancel and release their magnetic energy when they collide with magnetic flux of opposite polarity, or diffuse into the "empty" photosphere. We assume that cancellation of magnetic flux in collisions causes "flares" and determine the released energy as the difference in the square of the magnetic field flux $\left(E \sim B^{2}\right)$. The statistics of the simulated flares follow a power-law distribution in energy, $f(E) \sim E^{-a}$, where $a=2.2 \pm 0.1$. The size distribution function of the simulated active regions exhibits a power-law behavior with index $k \approx 1.93 \pm 0.08$, and the fractal dimension of the magnetized areas on the simulated solar surface is close to $D_{F} \sim 1.42 \pm 0.12$. Both quantities, $D_{F}$ and $k$, are inside the range of the observed values.
\end{abstract}

Subject headings: Sun: activity — Sun: flares — Sun: magnetic fields — Sun: photosphere

\section{INTRODUCTION}

Many solar phenomena, such as coronal heating and solar flares, are closely related to the evolution of active regions. Active regions are interpreted in this article as domains of strong magnetic field on the solar surface. The appearance of active regions on the solar surface is the result of the complex interplay between the buoyant forces and the turbulent convection zone working in the solar interior (Parker 1979). Convection zone dynamics have been studied numerically (Nordlund, Stein, \& Brandenburg 1996), but the magnetoconvection still remains in its infancy, and many questions remain unanswered (see review by Weiss 1997).

Many models have been suggested for the formation and evolution of active regions, such as the rise of a kink-unstable magnetic flux tube (Moreno-Insertis 1992) or the statistical description of the dynamical evolution of large-scale, twodimensional, fibril magnetic fields (Bogdan \& Lerche 1985). Schrijver et al. (1997) assumed that the thin flux tubes that constitute the active region move and interact only in the intergranular lanes and, in this way, form a fractal pattern. Several models have been developed using the anomalous diffusion of magnetic flux in the solar photosphere in order to explain the fractal geometry of the active regions (Lawrence 1991; Schrijver et al. 1992; Lawrence \& Schrijver 1993; Milovanov \& Zelenyj 1993). Lastly, a percolation model was used to simulate the formation and evolution of active regions (Wentzel \& Seiden 1992; Seiden \& Wentzel 1996), which models the evolution of active regions by reducing all the complicated solar MHD and turbulence to three dimensionless parameters. This percolation model explains the observed size distribution of active regions and their fractal characteristics (Meunier 1999).

Numerous observational studies have investigated the statistical properties of active regions, using full-disk magnetograms and $\mathrm{Ca}$ II plage region observations from the Mount Wilson Observatory and from the National Solar Observatory (see review by Howard 1996). These studies have examined among other parameters the size distribution of active regions and their fractal dimension: The size distribution function of the newly formed active regions exhibits a well-defined power law with index $\approx-1.94$, and active regions cover only a small fraction of the solar surface (around $\sim 8 \%$; Harvey 1993; Harvey $\&$ Zwaan 1993). The fractal dimension of the active regions has been studied using high-resolution magnetograms by Balke et al. (1993) and, more recently, by Meunier (1999). These authors found, not always using the same method, a fractal dimension $D_{F}$ in the range $1.3<D_{F}<1.8$.

In this article, we model the emergence and evolution of magnetic flux on the solar surface using a two-dimensional cellular automaton (CA), following techniques developed initially by Seiden \& Wentzel (1996). The dynamics of this automaton is probabilistic and is based on the competition between two "fighting" tendencies: stimulated or spontaneous emergence of new magnetic flux, and the disappearance of flux due to diffusion (i.e., dilution below observable limits), together with random motion of the flux tubes on the solar surface. The basic new element that we add to the Seiden \& Wentzel (1996) model is that we keep track of the energy release through flux cancellation (reconnection) if flux tubes of opposite polarities collide. We concentrate our analysis on the newly formed active regions. The old active regions undergo more complicated evolution; the dipoles are participating in large-scale flows associated with differential rotation and meridional flow (see Leighton 1964; Wang \& Sheeley 1994).

\section{THE MODEL}

We propose in this article that the main physical properties, as derived from the observations of the evolving active regions, can be summarized in simple CA rules. A two-dimensional quadratic grid with $200 \times 1000$ cells (grid sites) is constructed, in which each cell has four nearest neighbors. The grid is 
assumed to represent a large fraction of the solar surface. Initially, a small, randomly chosen percentage $(1 \%)$ of the cells is magnetized (loaded with flux) in the form of positively $(+1)$ and negatively $(-1)$ magnetized pairs (dipoles); the rest of the grid points are set to zero. Positive and negative cells evolve independently after their formation, but their percentage remains statistically equal.

The dynamical evolution of the model is controlled by the following probabilities:

1. $P$ is the probability that a magnetized cell is stimulating the appearance of new flux at one of its nearest neighbors. Each magnetized cell can stimulate its neighbors the first time step of its life only. This procedure simulates the stimulated emergence of flux that occurs as a result of the observed tendency of magnetic flux to emerge in regions of the solar surface where magnetic flux had previously emerged.

2. The flux of each magnetized cell has a probability $D_{m}$ to move to a random neighboring cell, simulating motions forced by the turbulent dynamics of the underlying convection zone. If the moving flux meets oppositely polarized flux in a neighboring cell, the fluxes cancel (through reconnection), giving rise to a "flare." If equal polarities meet in a motion event, the fluxes simply add up.

3. $D$ is the probability that a magnetized cell is turned into a nonmagnetized cell in one time step if it is next to nonmagnetized cells. This rule simulates the disappearing of flux below observational limits as a result of dilution caused by diffusion into the empty neighborhood.

4. $\varepsilon$ is the probability that a nonmagnetized cell is turned into magnetized cell spontaneously, independently of its neighbors, simulating the observed spontaneous emergence of new flux. Every newly appearing flux tube is accompanied by an oppositely polarized mate, taking into account the fact that flux always appears in the form of dipoles.

A detailed discussion of the connection between the parameters $P, D_{m}$, and $\varepsilon$ and the physical mechanisms acting in the evolution of active regions was established in the articles of Wentzel \& Seiden (1992) and Seiden \& Wentzel (1996).

\section{RESULTS}

The parameters used for the results reported here are $P=$ $0.185, D=0.005, D_{m}=0.05$, and $\varepsilon=10^{-6}$. They are chosen such that when following the evolution of our model and recording the percentage of magnetized cells, we find that it takes around 1000 time steps before the percentage of active cells is stabilized to a value that is close to the observed one (around $8 \%$ ). The size distribution of the simulated active regions can be approximated by a power-law fit of the form $N(s) \sim s^{-k}$, with $k=1.93 \pm 0.08$. Finally, we estimate the fractal dimension $D_{F}$ of the set of magnetized cells with the box-counting algorithm (e.g., Falconer 1990), finding $D_{F}=1.42 \pm 0.12$.

The cancellation of magnetic flux due to collisions of oppositely polarized magnetic flux tubes in motion events leads to the release of energy, whose amount we assume to be proportional to the difference in the square of the magnetic flux before and after the event. In Figure $1 a$, we plot the released energy $E(t)$ as a function of time. Figure $1 b$ shows the energy distribution of the recorded "flares": It follows a power law, $f(E) \sim E^{-a}$, with $a=2.2 \pm 0.1$, for energies $E>20$. For $E<20$, finite-resolution effects must be expected to bias the distribution, so we do not draw conclusions for the small energies. It is important to note also that the power law in the

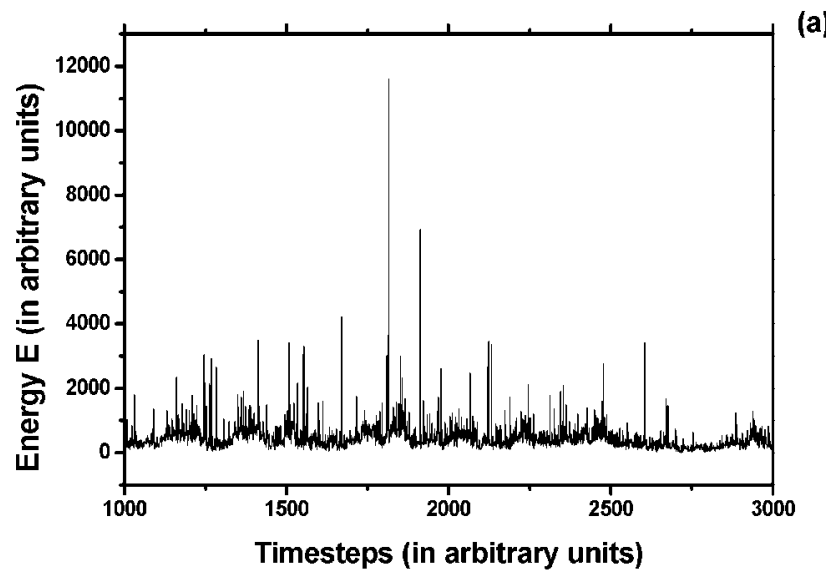

(a)

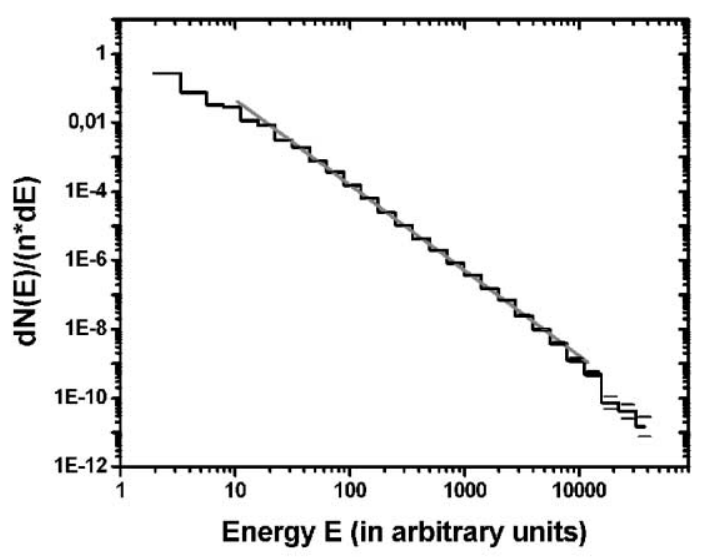

(b)

FIG. 1.-(a) Energy released in the cancellation of magnetic flux as a function of time, using the parameters $P=1.185, D=0.005, D_{m}=0.05$, and $\varepsilon=10^{-6}$. (b) Energy distribution of the recorded flares. The power-law index is $2.2 \pm 0.1$.

distribution of energy extends over three decades. In the top panel of Figure 2, we present a small portion of the grid. The dark areas correspond to negative polarity, and the bright ones to positive polarity. The spatial locations of the flares are marked with circles, with the size of the circles proportional to the logarithm of the locally released energy.

A variation of the parameters $P, D$, and $D_{m}$ does not alter the power-law behaviors or the fractality; they seem to be $g e$ neric properties of the model. The exact values of the powerlaw indices $k, a$, and $D_{F}$ depend, though, on the free parameters but remain inside the observed limits, even for a large variation of the parameters $P, D$, and $D_{m}$. The results are also independent of $\varepsilon$ as long as it remains small enough. In several independent simulations, we used different values for the stimulation probability $P$, keeping $D$ and $D_{m}$ constant, and found that $k$ and $a$ are closely correlated (see Fig. 2, bottom).

\section{SUMMARY AND DISCUSSION}

We have introduced a probabilistic CA to explain the formation and evolution of active regions and the associated energy release. The rules used simulate the competition between the stimulated emergence of new magnetic flux and the gradual 

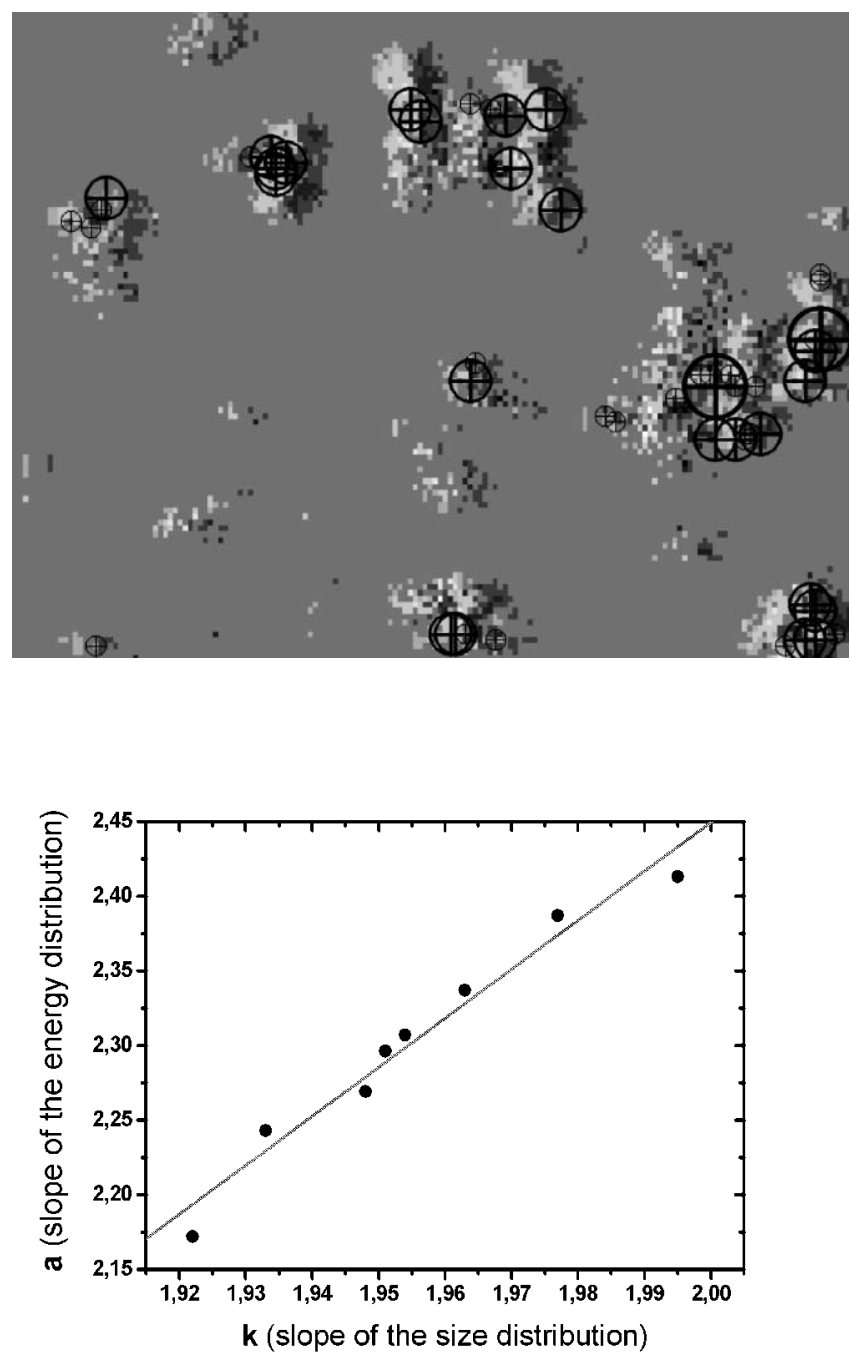

FIG. 2.-Top panel: Small portion of the modeled grid. The dark areas represent negative magnetic flux, and the white ones positive magnetic flux. The explosions (flares) appear randomly at the interface of regions of oppositely polarized magnetic flux. The circles represent the positions of the flares, with their radius being proportional to the logarithm of the released energy. In the bottom panel, there is a linear dependence of the size distribution active region index $k$ with the energy release index $a$ when the stimulation probability varies $0.180<P<1.87, D=0.005, D_{m}=0.05$, and $\varepsilon=10^{-6}$.

diffusion, as well as the motion of flux across the photosphere. We have compressed all the complicated physics involved in the formation of active regions into three dimensionless parameters, the probabilities $P, D$, and $D_{m}$, of which actually only $P$ and $D_{m}$ play a decisive, major role in the results. The fourth parameter, $\varepsilon$, plays no significant role in the evolution of the model (see $\S 2$ ).

Our main results are as follows: (1) The model yields a power-law distribution of the active region sizes, and the active regions form a fractal set. (2) The collision and cancellation of oppositely polarized magnetic flux cause flares. The energy distribution of the flares follows a power-law distribution with an exponent $a=2.2 \pm 0.1$. By varying the parameters, the power law is conserved, and just the power-law index changes. (3) The flares are distributed along the lines where regions of opposite flux meet (see Fig. 2, top). (4) Using different values for the stimulation probability $P$, we find a close correlation between the size distribution exponent $k$ and the energy release index $a$ (see Fig. 2, bottom).

The results derived from our model concerning the size distribution and the fractal dimension are in quantitative and qualitative agreement with the existing observations and with the results of the percolation models proposed previously (Seiden \& Wentzel 1996; Meunier 1999). The new result of our model is basically the connection of photospheric dynamics to energy release. This primary energy release through magnetic reconnection may well be the basic physical cause for the various types of observed low atmosphere emission events, such as coronal bright points (Parnell \& Jupp 2000; Krucker \& Benz 1998), X-ray network flares (Krucker et al. 1997), transition region impulsive EUV emission (Benz \& Krucker 2002), and $\mathrm{H} \alpha$ bright points (Ellerman bombs; Georgoulis et al. 2002). All these emissions may play a role in coronal heating (this point is still under strong debate), and in particular it was recently claimed that impulsive, chromospheric EUV emissions are important signatures of not just the coronal heating process but also of the supply of mass to the corona (Brown et al. 2000). It is interesting to note that all of the mentioned observed explosive phenomena exhibit power-law distributions in energy, with slopes roughly in the range between 1.5 and 2.5, coinciding with what we find here for the primary photospheric energy release. We believe that the observed discrepancies in the indices (especially on $a$ ) are directly related to the dynamical evolution of the active regions. The predicted correlation between the power-law indices of the distributions of the released energy and of the sizes of active regions can be checked in existing observations. These results may stimulate a new explanation for the statistical properties of impulsive solar energy release events in the low atmosphere. We believe that CA models, when used in conjunction with MHD numerical codes, can become a valuable tool for the study of the statistical properties of active regions.

We thank our colleague A. Anastasiadis for making several suggestions and comments on the manuscript.

\section{REFERENCES}

Balke, A. C., Schrijver, C. J., Zwaan, C., \& Tarbell, T. D. 1993, Sol. Phys., 143,215

Benz, A. O., \& Krucker, S. 2002, ApJ, 568, 413

Bogdan, T. J., \& Lerche, I. 1985, ApJ, 296, 719

Brown, J. C., Krucker, S., Güdel, M., \& Benz, A. O. 2000, A\&A, 359, 1185

Falconer, K. 1990, Fractal Geometry (Chichester: Wiley)

Georgoulis, M. K., Rust, D. M., Bernasconi, P. N., \& Schmieder, B. 2002, ApJ, 575, 506

Harvey, K. L. 1993, Ph.D. thesis, Univ. Utrecht

Harvey, K. L., \& Zwaan, C. 1993, Sol. Phys., 148, 85

Howard, R. F. 1996, ARA\&A, 34, 75

Krucker, S., \& Benz, A. O. 1998, ApJ, 501, L213

Krucker, S., Benz, A. O., Bastian, T. S., \& Acton, L. W. 1997, ApJ, 488, 499

Lawrence, J. K. 1991, Sol. Phys., 135, 249
Lawrence, J. K., \& Schrijver, C. J. 1993, ApJ, 411, 402

Leighton, R. B. 1964, ApJ, 140, 1547

Meunier, N. 1999, ApJ, 515, 801

Milovanov, A. V., \& Zelenyj, L. M. 1993, Phys. Fluids. B, 5(7), 2609

Moreno-Insertis, F. 1992, in Sunspots: Theory and Observations, ed. J. H. Thomes \& N. O. Weiss (Dordrecht: Kluwer), 385

Nordlund, Å., Stein, R. F., \& Brandenburg, A. 1996, Bull. Astron. Soc. India, 24, 261

Parker, E. N. 1979, Cosmical Magnetic Fields (Oxford: Clarendon)

Parnell, C. E., \& Jupp, P. E. 2000, ApJ, 529, 554

Seiden, P. E., \& Wentzel, D. G. 1996, ApJ, 460, 522

Schrijver, C. J., Title, A. M., Hagenaar, H. J., \& Shine, R. A. 1997, Sol. Phys., 175,329 
Schrijver, C. J., Zwaan, C., Balke, A. C., Tarbell, T. D., \& Lawrence, J. K. 1992, A\&A, 253, L1

Wang, Y. M., \& Sheeley, N. 1994, ApJ, 430, 399
Weiss, N. 1997, in Advances in the Physics of Sunspots, ed. B. Schmieder, J. C. del Toro Iniesta, \& M. Vazquez (Dordrecht: Kluwer), 21 Wentzel, D. G., \& Seiden, P. E. 1992, ApJ, 390, 280 